PROCEEDINGS OF THE

AMERICAN MATHEMATICAL SOCIETY

Volume 134, Number 2, Pages 541-548

S 0002-9939(05)08000-7

Article electronically published on July 18, 2005

\title{
ON THE DETERMINATION OF A MEASURE BY THE ORBITS GENERATED BY ITS LOGARITHMIC POTENTIAL
}

\author{
DIMITRIOS BETSAKOS AND SIMELA GRIGORIADOU
}

(Communicated by Juha M. Heinonen)

\begin{abstract}
We say that a logarithmic potential generates a curve in the plane if a unit mass traces the curve under the action of the potential. We consider the following problem: A one-parameter family of plane curves is given. We assume that these curves lie in the complement of a compact set $K$. Find all measures supported in $K$ whose potentials generate each of the given curves. We solve this problem when $K$ is the unit circle in three specific cases: (a) when the given curves are straight lines through the origin, (b) when the curves are straight lines through a point on the unit circle, and (c) when the curves are circles centered at the origin. The solution involves the Poisson integral and its boundary behavior.
\end{abstract}

\section{An inVerse problem in potential theory}

Let $\sigma$ be a finite Borel measure with support in a compact set $K$ that lies in the plane $\mathbb{R}^{2}$. For notational convenience we identify $\mathbb{R}^{2}$ with the complex plane $\mathbb{C}$. The logarithmic potential of $\sigma$ is the function $V_{\sigma}: \mathbb{C} \rightarrow(-\infty, \infty]$ defined by

$$
V_{\sigma}(z)=\int_{K} \log \frac{1}{|z-\zeta|} d \sigma(\zeta) .
$$

It is well known (see e.g. [6, Ch.3]) that $V_{\sigma}$ is a function superharmonic in $\mathbb{C}$ and harmonic in the complement of its support (which contains the set $\mathbb{C} \backslash K$ ).

Let $\alpha$ be an orbit in the plane. By orbit here we mean a $C^{2}$ function $\alpha(t)=$ $x(t)+i y(t): I \rightarrow \mathbb{C}$ defined on a closed interval $I \subset \mathbb{R}$. A curve is a set of points $x+i y \in \mathbb{C}$ that satisfy an equation of the form $\mathrm{f}(x, y)=q$, where $q$ is a real number and $\mathrm{f}$ is a $C^{2}$ real function defined on an open subset of $\mathbb{C}$. By the usual abuse of language, we will say that an orbit $\alpha$ lies in a set $A \subset \mathbb{C}$ if $\alpha(t) \in A$ for all $t \in I$. Of course every orbit $\alpha$ lies on the curve $\{\alpha(t): t \in I\}$ which is the trace of $\alpha$. The equation $\mathrm{f}(x, y)=q$ of the trace can be found (in principle) by eliminating $t$ from the equations $x=x(t), y=y(t)$.

We say that the potential $V_{\sigma}$ generates the orbit $\alpha$ if Newton's second law holds:

$$
\frac{d^{2} \alpha}{d t^{2}}(t)=\frac{d^{2} x}{d t^{2}}(t)+i \frac{d^{2} y}{d t^{2}}(t)=-\frac{\partial V_{\sigma}}{\partial x}(\alpha(t))-i \frac{\partial V_{\sigma}}{\partial x}(\alpha(t)), \quad t \in I .
$$

Received by the editors May 28, 2004 and, in revised form, September 30, 2004.

2000 Mathematics Subject Classification. Primary 31A15; Secondary 31A05, 70F99, 70K99.

Key words and phrases. Logarithmic potential, inverse problem, reflection principle, harmonic function, Poisson integral. 
Physically this means that a unit point mass placed on a point $\alpha\left(t_{o}\right)$ of $\alpha$ with velocity $\alpha^{\prime}\left(t_{o}\right)$ traces the orbit $\alpha$ under the attraction of a mass that lies on the cylinder $K \times \mathbb{R}$, and its distribution on every plane parallel to the $x y$-plane is $\sigma$. More details about the physical interpretation of logarithmic potentials can be found in [4, Chapter III]. We say that the potential $V_{\sigma}$ generates the curve $\mathbf{f}(x, y)=q$ if it generates an orbit lying on this curve.

The problem we wish to study is the following:

Problem 1. Let $K$ be a compact set in $\mathbb{C}$ and let $\mathcal{F}=\{\mathrm{f}(x, y)=p: p \in \mathbb{R}\}$ be a one-parameter family of curves, all lying in $\mathbb{C} \backslash K$. Find all measures $\sigma$ in $K$ whose logarithmic potentials generate each of the curves of $\mathcal{F}$.

A related problem, the inverse problem of dynamics, has been studied recently. We refer the reader to the paper [2] by G. Bozis for a survey on this problem and its history. A special case of this problem is the following: Suppose that $\mathcal{F}=\{\mathrm{f}(x, y)=p: p \in \mathbb{R}\}$ is a one-parameter family of curves in the plane. Find all $C^{1}$ functions $V$ (not necessarily logarithmic potentials) that generate each of the curves of $\mathcal{F}$. Bozis (see [1, 2] and the references therein) proved that if a $C^{2}$ function $V$ is a solution of the above problem, then it satisfies the second-order partial differential equation

$$
\frac{\partial^{2} V}{\partial x^{2}}-\frac{\partial^{2} V}{\partial y^{2}}-\kappa \frac{\partial^{2} V}{\partial x \partial y}+\lambda \frac{\partial V}{\partial x}+\mu \frac{\partial V}{\partial y}=0
$$

where

$$
\kappa=\frac{1-\gamma^{2}}{\gamma}, \quad \lambda=\frac{1}{\gamma \Gamma}\left(\frac{\partial \Gamma}{\partial y}-\gamma \frac{\partial \Gamma}{\partial x}\right), \quad \mu=\lambda \gamma+\frac{3 \Gamma}{\gamma},
$$

and the functions $\gamma(x, y)$ and $\Gamma(x, y)$ are given by

$$
\gamma=\frac{\partial \mathrm{f}}{\partial y}\left(\frac{\partial \mathbf{f}}{\partial x}\right)^{-1} \text { and } \Gamma=\gamma \frac{\partial \gamma}{\partial x}-\frac{\partial \gamma}{\partial y}
$$

In fact (1.3) holds when the given family is not a family of straight lines. If the given family consists of straight lines (i.e. when $\Gamma(x, y)=0$ ), then every function $V$ that generates them must satisfy the first-order partial differential equation

$$
\frac{\partial V}{\partial x}+\gamma \frac{\partial V}{\partial y}=0
$$

Returning to Problem 1, we see that if the measure $\sigma$ is one of its solutions, then its potential must satisfy (1.3) or (1.6). Using (1.1) and differentiation under the integral sign (provided, of course, that such an operation can be justified), we arrive at an integral equation. This equation must be satisfied by every measure $\sigma$ supported on $K$ and generating each of the curves of the family $\mathcal{F}$.

In this paper we study Problem 1 under the additional assumption that $\sigma$ is supported in the unit circle $\mathbb{T}$, that is, $K=\mathbb{T}$. Under this assumption, we solve the problem in three cases:

Case (a): The given family consists of linear segments on lines passing through the origin. This case is studied in Section 4. We find that the measure $\sigma$ must be uniformly distributed on $\mathbb{T}$.

Case (b): The given family consists of linear segments on lines passing through the point $1 \in \mathbb{T}$. This case is studied in Section 5 . We find that if the observed segments are inside $\mathbb{T}$, then $\sigma$ must be the sum of a uniform distribution on $\mathbb{T}$ and 
a Dirac measure on 1 , while if the observed segments are outside $\mathbb{T}$, then $\sigma$ must be a Dirac measure on 1 .

Case (c): The given family consists of circular arcs lying on circles centered at the origin. This case is studied in Section 6 . We find that $\sigma$ must be uniformly distributed on $\mathbb{T}$.

The proof of the above results involves various properties of harmonic functions. A reflection principle (Section 2) is used to prove in Section 3 that if a logarithmic potential generates a linear segment, then it must be locally symmetric with respect to this segment. We also use the Poisson integral and its boundary behavior.

\section{A REFLECTION PRINCIPLE FOR HARMONIC FUNCTIONS}

In this section we prove a form of the reflection principle for harmonic functions (Theorem 2 below). This result is certainly known, but we prove it for the sake of completeness. We will need some new pieces of notation. If $z=x+i y \in \mathbb{C}$, then by $\hat{z}$ we denote the reflection of $z$ in the real axis: $\hat{z}=x-i y$; if $A \subset \mathbb{C}$, then $\widehat{A}=\{\hat{z}: z \in A\}$. Below we will use some results related to the Dirichlet problem; for these results we refer to $[\underline{6}$.

Theorem 1. Let $G$ be a bounded plane domain, regular for the Dirichlet problem and symmetric with respect to the real axis. Let $f$ be a bounded, continuous, real function on $\partial G$ with $f(\zeta)=f(\hat{\zeta})$ for all $\zeta \in \partial G$. Let $u$ be the harmonic function in $G$ with $u=f$ on $\partial G$. Then

$$
u(z)=u(\hat{z}), \forall z \in G,
$$

and

$$
\frac{\partial u}{\partial y}(x)=0, \forall x \in G \cap \mathbb{R}
$$

Proof. Let $v(z)=(u(z)+u(\hat{z})) / 2$. Then $v(z)=v(\hat{z}), z \in G$, and

$$
\lim _{z \rightarrow \zeta} v(z)=f(\zeta), \forall \zeta \in \partial G .
$$

Therefore $u=v$ and (2.1) is proved. Also, because of (2.1),

$$
\frac{\partial u}{\partial y}(x)=\lim _{h \rightarrow 0} \frac{u(x+i h)-u(x-i h)}{2 h}=0, \forall x \in G \cap \mathbb{R},
$$

and (2.2) is proved.

Theorem 2. Let D be a bounded plane domain, regular for the Dirichlet problem. Assume that $D$ lies in the upper half-plane and that the boundary of $D$ contains an open subset $\tau$ of $\mathbb{R}$. Let $u$ be a function harmonic in $D$ and continuous on the closure of $D$. If $\frac{\partial u}{\partial y}(x)=0$ for all $x \in \tau$, then the function

$$
u_{1}(z)=\left\{\begin{array}{l}
u(z), z \in D \cup \tau, \\
u(\hat{z}), z \in \widehat{D}
\end{array}\right.
$$

is harmonic in $D \cup \tau \cup \widehat{D}$.

Proof. Let $v$ be the harmonic function in $G:=D \cup \tau \cup \widehat{D}$ with boundary values

$$
v(z)=\left\{\begin{array}{l}
u(\zeta), \zeta \in(\partial G \cap\{z: \operatorname{Im} z \geq 0\}), \\
u(\hat{\zeta}), \zeta \in \partial G \cap\{z: \operatorname{Im} z<0\} .
\end{array}\right.
$$


Then, by Theorem $1 \frac{\partial v}{\partial y}=0$ on $\tau$. Therefore the function $h=u-v$ is the solution of the Dirichlet-Neumann problem in $D$ with boundary conditions $h=0$ on $\partial D \backslash \tau$, $\frac{\partial h}{\partial y}=0$ on $\tau$. By the uniqueness of the solution (see [5, Chapter 2, Theorem 9]), we have $v(z)=u(z), \forall z \in D$. By the same argument, $v(z)=u(\hat{z}), \forall z \in \widehat{D}$. Both $v$ and $u_{1}$ are continuous in $G$. Hence $v=u_{1}$ in $G$ and the theorem is proved.

\section{Potentials generating a straight line}

In this section we prove the following theorem.

Theorem 3. Let $\sigma$ be a finite Borel measure with compact support $K \subset \mathbb{C}$. Suppose that the logarithmic potential $V_{\sigma}$ of $\sigma$ generates an orbit $\alpha$ that lies in a straight line c. Then $V_{\sigma}$ is locally symmetric with respect to $c$ in the sense that $V_{\sigma}(z)=V_{\sigma}(\hat{z})$ for all $z$ in a neighborhood of the trace of $\alpha$ ( $\hat{z}$ is the reflection of $z$ in $c$ ).

Proof. Without loss of generality we assume that $c=\mathbb{R}$. Then the orbit $\alpha$ has a formula of the form $\alpha(t)=x(t) \in \mathbb{R}, t \in[a, b]$. We fix $t_{o} \in[a, b]$ and set $x_{o}=x\left(t_{o}\right)$. Let $G$ be a disk of center $x_{o}$ and radius small enough so that $G \cap \mathbb{R} \subset\{x(t): t \in$ $[a, b]\}$ and $G \subset \mathbb{C} \backslash K$. Since $V_{\sigma}$ generates the orbit $\alpha$, we have

$$
\frac{\partial V_{\sigma}}{\partial y}(x(t))=0, t \in[a, b] .
$$

Hence $\frac{\partial V_{\sigma}}{\partial y}(x)=0, \forall x \in \mathbb{R} \cap G$. We consider the function $u$ defined as follows:

$$
u(z)=\left\{\begin{array}{l}
V_{\sigma}(z), z \in G \cap\{z: \operatorname{Im} z \geq 0\} \\
V_{\sigma}(\hat{z}), z \in G \cap\{z: \operatorname{Im} z<0\} .
\end{array}\right.
$$

By Theorem 2, $u$ is harmonic in $G$. Since $u$ coincides with $V_{\sigma}$ in an open subset of $G$, we have $u=V_{\sigma}$ in $G$ (by the identity principle for harmonic functions; see [6). Hence $V_{\sigma}(z)=V_{\sigma}(\hat{z})$ for all $z \in G$. By a compactness argument, this equality holds for all $z$ in a neighborhood of the trace of $\alpha$.

\section{Straight Lines Passing through the origin}

From now on we consider a finite Borel measure $\sigma$ whose support is a subset of the unit circle $\mathbb{T}$, and we suppose that it generates each curve of a one-parameter family of curves. Our aim will be to determine the measure $\sigma$.

In this section the observed orbits lie on straight lines through the origin. More precisely, let $D$ be an open disk in $\mathbb{C} \backslash \mathbb{T}$, let $\left\{c_{p}: p \in J\right\}$ be the family of all straight lines that pass through the origin and intersect $D$, and let $s_{p}=c_{p} \cap D$ (the parameter $p$ takes values in a suitable open interval $J$ ). If a measure on $\mathbb{T}$ generates the linear segments $s_{p}$, then it must be uniformly distributed on $\mathbb{T}$ :

Theorem 4. Suppose that a finite Borel measure $\sigma$ supported in $\mathbb{T}$ generates each linear segment $s_{p}$ in the disk $D$. Then for every Borel set $A \subset \mathbb{T}$, we have $\sigma(A)=$ $C \cdot \Lambda(A)$, where $\Lambda(A)$ is the Lebesgue measure (length) of $A$ and $C$ is a constant.

Proof. By Theorem 3, $V_{\sigma}$ is locally symmetric with respect to each line $c_{p}$. This implies that $V_{\sigma}$ is radial in $D$. Since $V_{\sigma}$ is also harmonic in $\mathbb{C} \backslash \mathbb{T}$, we have

$$
V_{\sigma}(z)=a \log \frac{1}{|z|}+b, \forall z \in D,
$$

where $a, b$ are real constants. Here we distinguish two cases. 
Case 1: $D \subset \mathbb{C} \backslash \mathbb{D}$.

By the identity principle for harmonic functions, (4.1) holds for all $z \in \mathbb{C} \backslash(\mathbb{D} \cup \mathbb{T})$. We write $z=r e^{i \theta} \in \mathbb{C} \backslash(\mathbb{D} \cup \mathbb{T}), \zeta=e^{i \varphi} \in \mathbb{T}$ and we compute the derivative $\frac{\partial V_{\sigma}}{\partial \theta}$ :

$$
\begin{aligned}
\frac{\partial V_{\sigma}}{\partial \theta}\left(r e^{i \theta}\right) & =\frac{\partial}{\partial \theta} \int_{0}^{2 \pi} \log \frac{1}{\left|r e^{i \theta}-e^{i \varphi}\right|} d \sigma(\varphi) \\
& =\int_{0}^{2 \pi} \frac{\partial}{\partial \theta} \log \frac{1}{\left|r e^{i \theta}-e^{i \varphi}\right|} d \sigma(\varphi) .
\end{aligned}
$$

The differentiation under the integral sign is justified by a standard application of Lebesgue's dominated convergence theorem; see e.g. [3, Theorem (2.27)]. A simple computation gives

$$
\frac{\partial}{\partial \theta} \log \frac{1}{\left|r e^{i \theta}-e^{i \varphi}\right|}=\frac{r \sin (\varphi-\theta)}{1+r^{2}-2 r \cos (\varphi-\theta)} .
$$

The equations (4.2) and (4.3) yield

$$
\begin{aligned}
\frac{\partial V_{\sigma}}{\partial \theta}\left(r e^{i \theta}\right) & =\int_{0}^{2 \pi} \frac{r \sin (\varphi-\theta)}{1+r^{2}-2 r \cos (\varphi-\theta)} d \sigma(\varphi) \\
& =\int_{0}^{2 \pi} \frac{\frac{1}{r} \sin (\varphi-\theta)}{1+\frac{1}{r^{2}}-2 \frac{1}{r} \cos (\varphi-\theta)} d \sigma(\varphi) .
\end{aligned}
$$

The function

$$
Q\left(\rho e^{i \theta}\right):=\frac{2 \rho \sin \theta}{1+\rho^{2}-2 \rho \cos \theta}, 0 \leq \rho<1, \theta \in[0,2 \pi],
$$

is the conjugate Poisson kernel. Therefore the function $\frac{\partial V_{\sigma}}{\partial \theta}$ is harmonic conjugate to the function

$$
u\left(r e^{i \theta}\right):=\pi P_{1 / r}[\sigma](\theta), \quad r>1, \theta \in[0,2 \pi]
$$

where

$$
P_{1 / r}[\sigma](\theta):=\frac{1}{2 \pi} \int_{0}^{2 \pi} \frac{1-\frac{1}{r^{2}}}{1+\frac{1}{r^{2}}-2 \frac{1}{r} \cos (\varphi-\theta)} d \sigma(\varphi)
$$

is the Poisson integral of the measure $\sigma$.

It follows from (4.1) that $\frac{\partial V_{\sigma}}{\partial \theta}=0$ in $\mathbb{C} \backslash(\mathbb{D} \cup \mathbb{T})$. Hence $P_{1 / r}[\sigma](\theta)$ is a real constant $C$ for $e^{i \theta} / r \in \mathbb{D}$. By the theory of the boundary behavior of Poisson integrals (see [7, Theorem 11.30]), we infer that $\sigma=C \cdot \Lambda$ on $\mathbb{T}$.

Case 2: $D \subset \mathbb{D}$.

By the identity principle for harmonic functions, (4.1) holds for all $z \in \mathbb{D} \backslash\{0\}$. But $V_{\sigma}$ is harmonic at the origin; hence $a=0$ and we conclude that $V_{\sigma}=b$, a constant, in $\mathbb{D}$. Now, as in Case 1 , we use the equality, $\frac{\partial V_{\sigma}}{\partial \theta}=0$, the Poisson integral and its conjugate, and conclude that $\sigma$ is a constant multiple of the Lebesgue measure on $\mathbb{T}$.

Remark 1. Another proof of the theorem above can be found by using the integral equation mentioned in the first section. The integral equation leads, after some calculations, to the equality

$$
\int_{0}^{2 \pi} \frac{r \sin (\varphi-\theta)}{1+r^{2}-2 r \cos (\varphi-\theta)} d \sigma(\varphi)=0,
$$

and from now on this new proof is the same as the proof above. 
Remark 2. It is interesting that $\sigma$ is determined by the observation of the line segments it generates in an arbitrarily small disk. Also this disk can be arbitrarily far away from the support of the measure $\sigma$.

Remark 3. We found that the logarithmic potential which generates the line segments $s_{p}$ is constant inside $\mathbb{T}$ and radial outside $\mathbb{T}$. We observe now that the orbits on $s_{p}$ cannot be arbitrary. For example there is no potential (of a measure supported on $\mathbb{T}$ ) that can generate orbits that lie on $s_{p}$ inside $\mathbb{T}$, and the velocity is not constant for each one of them.

Remark 4. From the proof of Theorem 4 we infer that if the logarithmic potential of a measure supported in $\mathbb{T}$ is constant in $\mathbb{D}$, then the measure is necessarily a constant multiple of the equilibrium measure of $\mathbb{T}$.

\section{Straight Lines PASsing Through the POINT 1}

In this section we consider a finite Borel measure $\sigma$ supported in the unit circle $\mathbb{T}$. Let $D$ be an open disk in $\mathbb{C} \backslash \mathbb{T}$, let $\left\{s_{p}: p \in J\right\}$ be the family of all straight lines that pass through the point 1 and intersect $D$, and let $c_{p}=s_{p} \cap D$ (the parameter $p$ takes values in a suitable open interval $J$ ).

Theorem 5. Suppose that a finite Borel measure $\sigma$ supported in $\mathbb{T}$ generates each linear segment $c_{p}$ in the disk $D$. If $D \subset \mathbb{D}$, then $\sigma=C_{1} \cdot \Lambda+C_{2} \cdot \delta_{1}$, where $C_{1}, C_{2}$ are constants and $\delta_{1}$ is the Dirac measure on 1 . If $D \subset \mathbb{C} \backslash(\mathbb{D} \cup \mathbb{T})$, then $\sigma=C_{3} \cdot \delta_{1}$, where $C_{3}$ is a constant.

Proof. Suppose first that $D \subset \mathbb{D}$. By Theorem 3. $V_{\sigma}$ is locally symmetric with respect to each line $s_{p}$. This implies that $V_{\sigma}(z)$ in $D$ depends only on the distance between $z$ and 1 . Since $V_{\sigma}$ is also harmonic in $\mathbb{D}$, we have

$$
V_{\sigma}(z)=C_{2} \log \frac{1}{|1-z|}+b, \forall z \in D,
$$

where $C_{2}, b$ are real constants. By the identity principle, (5.1) holds for all $z \in \mathbb{D}$.

Now consider the signed measure $\tau=\sigma-C_{2} \delta_{1}$. For $z \in \mathbb{D}$, we have

$$
V_{\tau}(z)=V_{\sigma}(z)-C_{2} V_{\delta_{1}}(z)=b .
$$

By the same arguments as in the proof of Theorem 4 (involving the Poisson integral), we conclude that $\tau$ has the form $C_{1} \cdot \Lambda$. Hence $\sigma=C_{1} \cdot \Lambda+C_{2} \cdot \delta_{1}$.

Next suppose that $D \subset \mathbb{C} \backslash(\mathbb{D} \cup \mathbb{T})$. Then, as above, we find that

$$
V_{\sigma}(z)=C_{3} \log \frac{1}{|1-z|}+C_{4}, \forall z \in \mathbb{C} \backslash(\mathbb{D} \cup \mathbb{T}),
$$

where $C_{3}, C_{4}$ are real constants. Consider the signed measure $\mu=\sigma-C_{3} \delta_{1}$. For $z \in \mathbb{C} \backslash(\mathbb{D} \cup \mathbb{T})$, we have

$$
V_{\mu}(z)=V_{\sigma}(z)-C_{3} V_{\delta_{1}}(z)=C_{4} .
$$

Again by the argument involving the Poisson integral, we conclude that $\mu$ has the form $C_{5} \cdot \Lambda$, where $C_{5}$ is a constant. Hence $\sigma=C_{5} \cdot \Lambda+C_{3} \cdot \delta_{1}$. Therefore

$$
V_{\sigma}(z)=2 \pi C_{5} \log \frac{1}{|z|}+C_{3} \log \frac{1}{|1-z|}+C_{6}, \quad z \in \mathbb{C} \backslash(\mathbb{D} \cup \mathbb{T}) .
$$

Comparing (5.5) with (5.3) we conclude that $C_{5}=0$. Hence $\sigma=C_{3} \cdot \delta_{1}$. 


\section{Circles Centered at the ORIGiN}

In this section we again consider a finite Borel measure $\sigma$ supported on the unit circle $\mathbb{T}$. Also let $\left\{s_{p}\right\}$ be the one-parameter family of circles centered at the origin. Let $D$ be a disk in $\mathbb{C} \backslash \mathbb{T}$ and let $c_{p}=s_{p} \cap D$ (the parameter $p$ takes values in a suitable open interval $J$ ). If $\sigma$ generates the circular $\operatorname{arcs} c_{p}$, then $\sigma$ is necessarily a constant multiple of the Lebesgue measure on $\mathbb{T}$ :

Theorem 6. Suppose that a finite Borel measure $\sigma$ supported on $\mathbb{T}$ generates each circular arc $c_{p}$ in the disk $D$. Then $\sigma=C \cdot \Lambda$, where $C$ is a constant.

Proof. We will assume that $D \subset \mathbb{D}$; the case $D \subset \mathbb{C} \backslash(\mathbb{T} \cup \mathbb{D})$ can be treated in an analogous way. We also assume without loss of generality that $\bar{D} \cap \mathbb{R}=\emptyset$ and $\bar{D} \cap \mathbb{T}=\emptyset$; otherwise we consider a smaller disk $D$.

The equation of the circles $s_{p}$ is $\mathrm{f}(x, y):=x^{2}+y^{2}=p ; p \in J$. For this $\mathrm{f}$, we compute the functions $\gamma, \Gamma, \kappa, \lambda, \mu$ using (1.5) and (1.4), and we find

$$
\begin{gathered}
\gamma=\frac{y}{x}, \Gamma=-\frac{y^{2}}{x^{3}}-\frac{1}{x}, \\
\kappa=\frac{x^{2}-y^{2}}{x y}, \lambda=\frac{3}{x}, \mu=-\frac{3}{y}, \quad(x, y) \in D .
\end{gathered}
$$

So, Bozis' equation (1.3) becomes

$$
\frac{\partial^{2} V_{\sigma}}{\partial x^{2}}-\frac{\partial^{2} V_{\sigma}}{\partial y^{2}}-\frac{x^{2}-y^{2}}{x y} \frac{\partial^{2} V_{\sigma}}{\partial x \partial y}+\frac{3}{x} \frac{\partial V_{\sigma}}{\partial x}-\frac{3}{y} \frac{\partial V_{\sigma}}{\partial y}=0,(x, y) \in D
$$

In this equation we substitute $V_{\sigma}$ from (1.1), differentiate under the integral sign (an operation justified by [3. Theorem (2.27)]), write $x+i y=r e^{i \theta} \in D, \zeta=e^{i \varphi} \in \mathbb{T}$, identify $\sigma$ with a measure on $[0,2 \pi]$, and (6.3), after some calculations, becomes

$$
\int_{0}^{2 \pi} \frac{\left[r^{2}+3-4 r \cos (\theta-\varphi)\right] \sin (\theta-\varphi)}{r \sin 2 \theta\left[r^{2}+1-2 r \cos (\theta-\varphi)\right]^{2}} d \sigma(\varphi)=0, r e^{i \theta} \in D .
$$

We set

$$
B(r, \theta, \varphi):=\frac{r \sin (\theta-\varphi)}{r^{2}+1-2 r \cos (\theta-\varphi)}
$$

and

$$
A(r, \theta)=\int_{0}^{2 \pi} B(r, \theta, \varphi) d \sigma(\varphi)
$$

After some calculations (6.4) becomes

$$
\frac{1}{r^{2} \sin 2 \theta} \int_{0}^{2 \pi}\left[2 B(r, \theta, \varphi)+r \frac{\partial B}{\partial r}(r, \theta, \varphi)\right] d \sigma(\varphi)=0, r e^{i \theta} \in D .
$$

Performing a differentiation under the integral sign (justified again by [3, Theorem $(2.27)]$ ), we see that $(\underline{6.5})$ is equivalent to

$$
2 A(r, \theta)+r \frac{\partial A}{\partial r}(r, \theta)=0, r e^{i \theta} \in D
$$

For each fixed $\theta,(6.6)$ is an ordinary linear differential equation in the variable $r$ which has the solutions

$$
A(r, \theta)=\frac{K(\theta)}{r^{2}}
$$


where $K(\theta)$ is an arbitrary function of $\theta$. But $A$ is harmonic function of $r e^{i \theta}$ in $D$ (it is the conjugate Poisson integral of $\sigma$ ). Using the Laplacian in polar coordinates we find that

$$
A(r, \theta)=\frac{c_{1} \cos \theta+c_{2} \sin \theta}{r^{2}}, r e^{i \theta} \in D,
$$

where $c_{1}, c_{2}$ are constants. By the identity principle this equality holds for all $r e^{i \theta} \in \mathbb{D} \backslash\{0\}$. Since $A$ is also harmonic at 0 , we have $c_{1}=c_{2}=0$ and therefore $A=0$ in $\mathbb{D}$. We continue as in the proof of Theorem 4. The Poisson integral of $\sigma$ must be equal to a real constant and therefore $\sigma$ must be a constant multiple of the Lebesgue measure on $\mathbb{T}$.

\section{REFERENCES}

1. G. Bozis, Szebehely's inverse problem for finite symmetrical material concentrations. Astron. Astrophys. 143 (1984), 360-364. MR0748011 (85d:70006)

2. G. Bozis, The inverse problem of dynamics: basic facts. Inverse problems 11 (1995), 637-708. MR 1345999 (96k:70004)

3. G.B. Folland, Real Analysis, Modern Techniques and Their Applications. Second Edition. Wiley 1999. MR1681462 (2000c:00001)

4. O.D. Kellogg, Foundations of Potential Theory. Dover Publications 1953.

5. M.H. Protter, H.F. Weinberger, Maximum Principles in Differential Equations. Springer-Verlag 1984. MR0762825 (86f:35034)

6. T. Ransford, Potential Theory in the Complex Plane. Cambridge Univ. Press 1995. MR.1334766 (96e:31001)

7. W. Rudin, Real and Complex Analysis. Third Edition. McGraw-Hill 1987. MR0924157 (88k:00002)

Department of Mathematics, Aristotle University of Thessaloniki, 54124 ThessaLONIKI, GREeCE

E-mail address: betsakos@math.auth.gr

Department of Mathematics, Aristotle University of Thessaloniki, 54124 ThessaLONIKI, GREECE 